\title{
Contexto de las hemorragias, en el puerperio inmediato
}

\author{
Delia Crespo Antepara, Lenin Byron Mendieta Toledo \\ Universidad de Guayaquil, Ecuador
}

Cómo referenciar este artículo/ How to reference this article:
Crespo Antepara D, Mendieta-Toledo LB. Contexto de las hemorragias, en el puerperio inmediato. Mem. Inst. Investig. Cienc. Salud. 2019; 17(3): 5-9

\section{RE S U M E N}

La hemorragia postparto es una de las complicaciones obstétricas más temidas por los obstetras a nivel mundial, pudiendo causar la muerte de las puérperas. El objetivo de este estudio fue determinar las causas de las hemorragias y las patologías asociadas a estas en las pacientes atendidas en el Hospital Gineco-Obstétrico Enrique C. Sotomayor de Guayaquil, Ecuador en el periodo 2016-2018 y que estaban en su puerperio inmediato. Las mujeres atendidas en ese periodo en dicha institución fueron 528, de las cuales 160 presentaron hemorragias en el puerperio inmediato. Las hemorragias por desgarro del suelo pélvico representaron un $48,75 \%$ y se asociaron en un $35 \%$ a hematomas en las paredes del suelo, las causadas por retención de restos placentarios, constituyeron un $26,25 \%$ y el $20 \%$ presentó acretismo placentario y finalmente las ocasionadas por atonía uterina fueron de un $25 \%$, correspondiendo un $25 \%$ a coagulopatías. Se concluyó que las hemorragias postparto por desgarro del suelo pélvico son la causa con mayor porcentaje en contraposición con otros estudios que indican que la atonía uterina es la causa más frecuente de hemorragias postparto inmediato.

Palabras clave: Hemorragia postparto, causas, patologías asociadas.

\section{Context of hemorrhages, in the immediate puerperium}

\begin{abstract}
A B S T R A C T
Postpartum hemorrhage is one of the obstetric complications most feared by obstetricians worldwide, and may cause the death of puerperal women. The objective of this study was to determine the causes of hemorrhages and the pathologies associated with these in the patients treated at the Enrique C. Sotomayor Obstetric Hospital of Guayaquil, Ecuador in the period 2016 - 2018 and that were in their immediate puerperium. The women who attended in that period were 528 women, 160 of them presented hemorrhages in the immediate puerperium. Hemorrhages by pelvic floor tearing represented $48.75 \%$ and $35 \%$ were associated to bruises in the floor walls, those caused by retention of placental remains constituted $26.25 \%$ and $20 \%$ presented accretism and finally those caused by uterine atony were $25 \%$ while $25 \%$ corresponded to coagulopathies. We concluded that the postpartum hemorrhages by tearing of the pelvic floor are the cause with the highest percentage; in contrast to other studies, which indicate that uterine aton is the most frequent cause of immediate postpartum hemorrhage.
\end{abstract}

Keywords: Postpartum hemorrhage, causes, associated pathologies.

\section{INTRODUCCIÓN}

La hemorragia postparto (HPP), es definida por la Organización Mundial de la Salud $(\mathrm{OMS})^{(1)}$ como "La pérdida de sangre de $500 \mathrm{ml}$ o más, en un periodo de 24 horas, posterior al parto"; esto en un parto normal y puede alcanzar hasta los 1000cc cuando es posterior a una cesárea, además, en un estudio realizado se señala que esta se presenta "entre el uno y el cinco por ciento de las mujeres con hemorragia posparto, y es más frecuente en partos por cesárea". La hemorragia puede producirse antes o después de la expulsión de la placenta. También, puede existir lo que se denomina hemorragia postparto secundaria, 
definida como cualquier hemorragia anormal o excesiva a través del canal de parto, que ocurre entre las primeras 24 horas y 12 semanas después del parto. Por lo tanto, este tipo de hemorragia puede conllevar a una inestabilidad hemodinámica del paciente, porque las pérdidas sanguíneas generalmente no pueden cuantificarse. De allí, que Pérez et al. ${ }^{(2)}$ también manifiesten que es una de las complicaciones obstétricas más temidas y sea una de las tres primeras causas de morbimortalidad materna a nivel mundial.

En países en desarrollo y en países económicamente desarrollados, la hemorragia postparto (HPP) es una de las principales causas de mortalidad y morbilidad materna severa. Aproximadamente 14 millones de mujeres sufren hemorragia post-parto anualmente. A nivel mundial, ocurren cada año 529.000 muertes relacionadas con el embarazo. La hemorragia postparto contribuye con 25-30\% de estas muertes en los países en desarrollo. En consecuencia, la hemorragia severa es la causa principal de mortalidad materna en el mundo ${ }^{(3,4)}$ Los determinantes y los factores de riesgo de HPP han sido estudiados para identificar a las mujeres embarazadas con riesgo aumentado. Varios artículos han citado determinantes de hemorragia post-parto, como el número de partos de las pacientes ${ }^{(5,6)}$, en nuestra región (América Latina y Caribe) los estudios realizados han sido observacionales tratando este tema ${ }^{(7)}$.

Asimismo, epidemiológicamente se estima que el $99 \%$ de las muertes relacionadas al embarazo, el parto o el puerperio, suceden en los países en vías de desarrollo ${ }^{(8)}$ siendo la hemorragia postparto la primera causa de muerte materna a nivel mundial; constituyendo según el informe del $25 \%$ del total de muertes maternas, en su mayoría en las primeras 24 horas postparto, por ser estas muy graves. De igual forma, en Ecuador, aproximadamente se presentan 34.000 defunciones al año por hemorragias postparto y de acuerdo al Instituto Nacional de Estadísticas y Censos del Ecuador ${ }^{(9)}$, en el 2015, la tasa de mortalidad materna fue de 49,2 por 100.000 nacidos vivos, siendo la hemorragia postparto una de las dos causas más frecuentes. De allí también, que hay que tomar en cuenta las causas, que conllevan a una hemorragia postparto. Ante esto, un estudio señala ${ }^{(10)}$ como causas más frecuentes: la atonía uterina, los traumas/laceraciones, la retención de retos placentarios post concepción y las alteraciones de la coagulación. Algunos autores consideran a la atonía uterina como la causa más frecuente ${ }^{(10,11)}$; responsable del 50 al $70 \%$ de las hemorragias post alumbramiento y del $4 \%$ de todas las muertes maternas. Esta pérdida del tono de la musculatura del útero trae como consecuencia la ausencia de contracciones, indispensables para la involución del útero tras el parto; pero su manejo preventivo puede realizarse durante trabajo de parto o después de este, con el uso de uteroinhibidores.

También, el desgarro del suelo pélvico es una causa a tomar en consideración, ya que el mismo va acompañado ${ }^{(12)}$ de una denervación de la musculatura del suelo pélvico por daño en los nervios pudendos y sacros, a consecuencia de partos vaginales, la cual pudo deberse a la instrumentación empleada en la atención del parto. Por lo tanto, hay que mencionar que la debilidad de los músculos que forman el suelo pélvico, es predisponente a que una mujer presente múltiple sintomatología. Igualmente, Pérez et al. ${ }^{(2)}$ refieren que su fisiopatología es multifactorial y la alteración de los músculos elevadores del ano y del núcleo fibroso del periné es el origen más frecuente de este problema; ocasionando desgarro del cérvix el más frecuente, seguido del vaginal.

En un mismo orden de ideas, podemos decir que dentro de la etiología de las hemorragias postparto según Pérez Barchilón et al. ${ }^{(2)}$ el manejo adecuado disminuye los eventos adversos derivados de la misma siendo la hemorragia postparto muy frecuente en el periodo de alumbramiento placentario, por lo que su extracción debe ser suave y mantenerse unida a la otra parte del cordón umbilical. Si la placenta no es expulsada espontáneamente en los 30 minutos después del parto, se habla de retención placentaria o en su defecto de una inserción anormal de la misma. En el primer caso, se procede a una exploración manual del útero y extracción de la placenta, la cual se efectúa introduciendo una mano enguantada en la cavidad uterina y controlando el fondo con la otra mano; se sigue el cordón umbilical hasta su inserción y se identifica el borde inferior de la placenta, para proceder a su separación mediante un movimiento de sierra.

De igual forma, hay que tomar en cuenta que al momento del parto las mujeres son susceptibles de poner en riesgo su vida ya que pueden sufrir una hemorragia. La mayoría de las hemorragias posparto no presentan factores de riesgo identificables, pero muchas de ellas pueden prevenirse con el manejo activo de la tercera etapa del parto (MATEP) ${ }^{(13)}$. 
Aunque en muchos países en desarrollo aún se utiliza el manejo expectante de la tercera etapa del parto se ha demostrado en la comparación en los estudios realizados por Bristol y Hinchingbrooke acerca del manejo activo de la tercera etapa del parto (MATEP) versus el manejo expectante o fisiológico de la tercera etapa del parto, que cuando se realizó el manejo activo la incidencia de hemorragia posparto fue significativamente menor $(5,9 \%$ con MATEP vs. $17,9 \%$ con manejo expectante; y $6,8 \%$ con MATEP vs. $16,5 \%$ sin MATEP) ${ }^{(13)}$.

Por todo lo mencionado anteriormente, se orientó esta investigación hacia el estudio de las hemorragias postparto, ya que estuvieron presentes en las mujeres atendidas en el Hospital Gineco-Obstétrico Enrique C. Sotomayor, Ecuador en el periodo enero 2016-2018, durante su puerperio inmediato. Se estableció como objetivo determinar las causas y consecuencias maternas de la hemorragia postparto en el puerperio inmediato y tener una visión de la magnitud del problema.

\section{MATERIALES Y MÉTODO}

Se realizó un estudio retrospectivo longitudinal, en las pacientes atendidas en el área de postparto del Hospital Gineco Obstétrico Enrique C. Sotomayor de enero 2016 - 2018, en Ecuador. Los documentos base fueron las historias clínicas de las pacientes atendidas en el postparto inmediato que presentaron hemorragias. Además, se sustentó el estudio con una revisión documental física y digital. El universo estuvo constituido por 528 pacientes atendidas por parto vaginal o por cesárea, de las cuales se incluyeron 160 pacientes: Por presentar hemorragia postparto inmediata, haber sido atendidas en el centro hospitalario, objeto de estudio, con evolución normal del embarazo y datos clínicos, obtenidos de las historias realizadas en el centro hospitalario, objeto de estudio.

Se solicitó el consentimiento del director del centro hospitalario, para la evacuación de la información y se firmó una carta de confidencialidad acogiendo el estudio a las normas éticas de Helsinki ${ }^{(14)}$, modificada en asamblea mundial en la $64^{a}$ Asamblea General, Fortaleza, Brasil, octubre 2013, la cual en su artículo 4 indica "El deber del médico es promover y velar por la salud, bienestar y derechos de los pacientes, incluidos los que participan en investigación médica..."; además, en su artículo 5 señala "El progreso de la medicina se basa en la investigación que, en último término, debe incluir estudios en seres humanos"; por último, en lo que ha esta investigación compete, el artículo 24 manifiesta "Deben tomarse toda clase de precauciones para resguardar la intimidad de la persona que participa en la investigación y la confidencialidad de su información personal".

\section{RESULTADOS}

Dentro de las causas maternas, la atonía uterina tuvo presente en 40 pacientes, el desgarro de suelo pélvico (canal) en 78 y la retención de restos de tejido placentario en 42 pacientes (Figura 1). Los resultantes maternos se presentan en desgarro de suelo pélvico con el más alto porcentaje, llegando a un $48,75 \%$ por sobre el $26,25 \%$ de retención de restos de tejido placentario y el $25 \%$ de casos de HPP por atonía.

Fuente: Hospital Gineco-Obstétrico Enrique C. Sotomayor, Ecuador (enero 2016-2018)

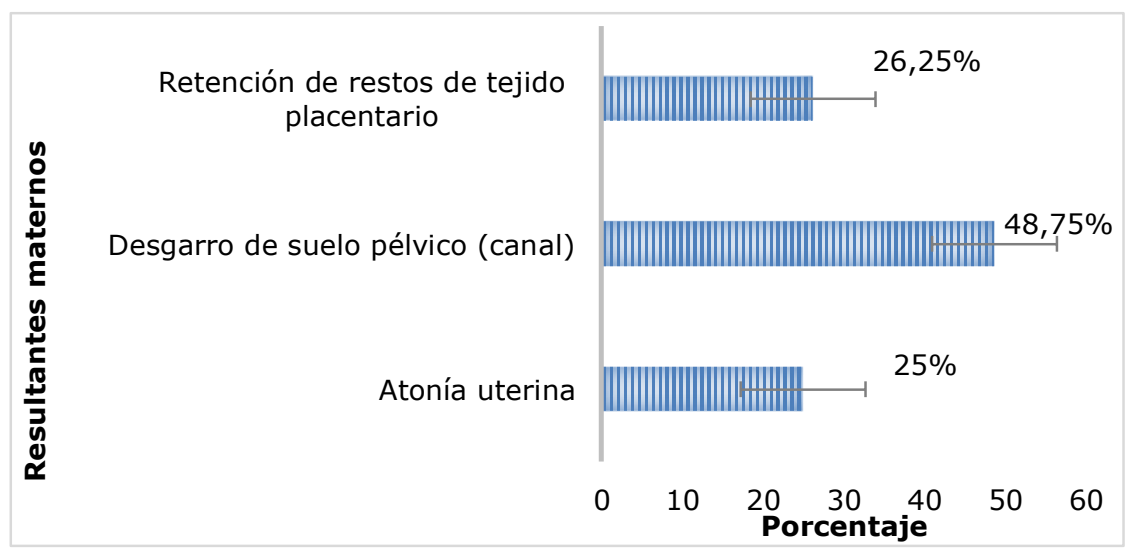

Figura 1: Principales causas de hemorragias postparto inmediato. 
En el estudio, existe un efecto colateral del desgarro del suelo pélvico. Con un $65 \%$ se evidencia en el estudio que no presentan hematomas, mientras que, el hematoma en las paredes del suelo pélvico se presenta en un $35 \%$ en las pacientes (Figura 2).

Fuente: Hospital Gineco-Obstétrico Enrique C. Sotomayor, Ecuador (enero 2016-2018)

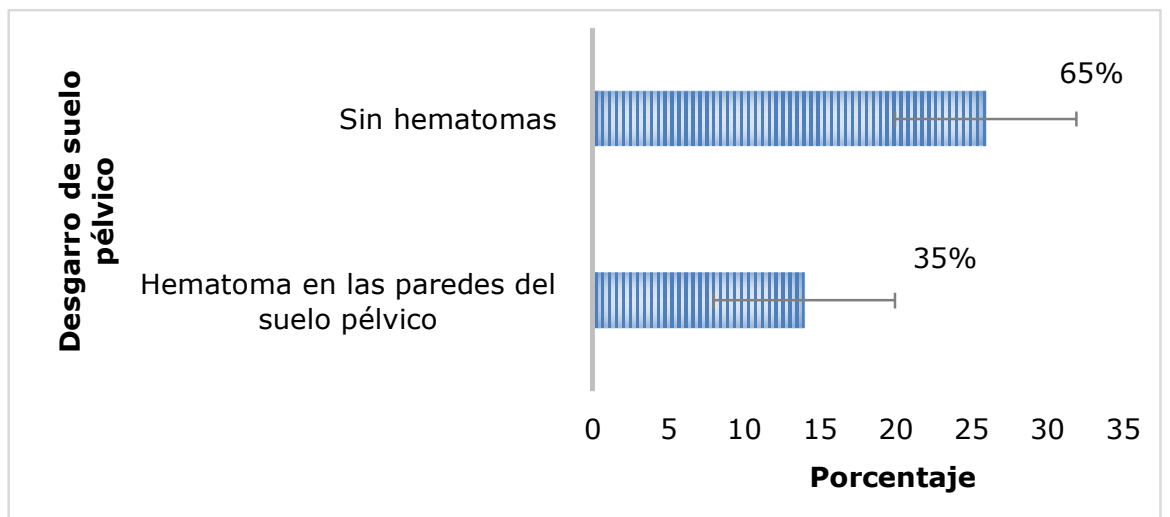

Figura 2: Patología asociada a la hemorragia postparto, por desgarro de suelo pélvico.

Los resultados por retención de restos placentarios se presentan sin complicaciones secundarias en un 55\%, mientras que, con acretismo existe un $20 \%$ de pacientes y con coagulopatías se presentaron un $25 \%$ de pacientes (Figura 3 ).

Fuente: Hospital Gineco-Obstétrico Enrique C. Sotomayor, Ecuador (enero 2016-2018)

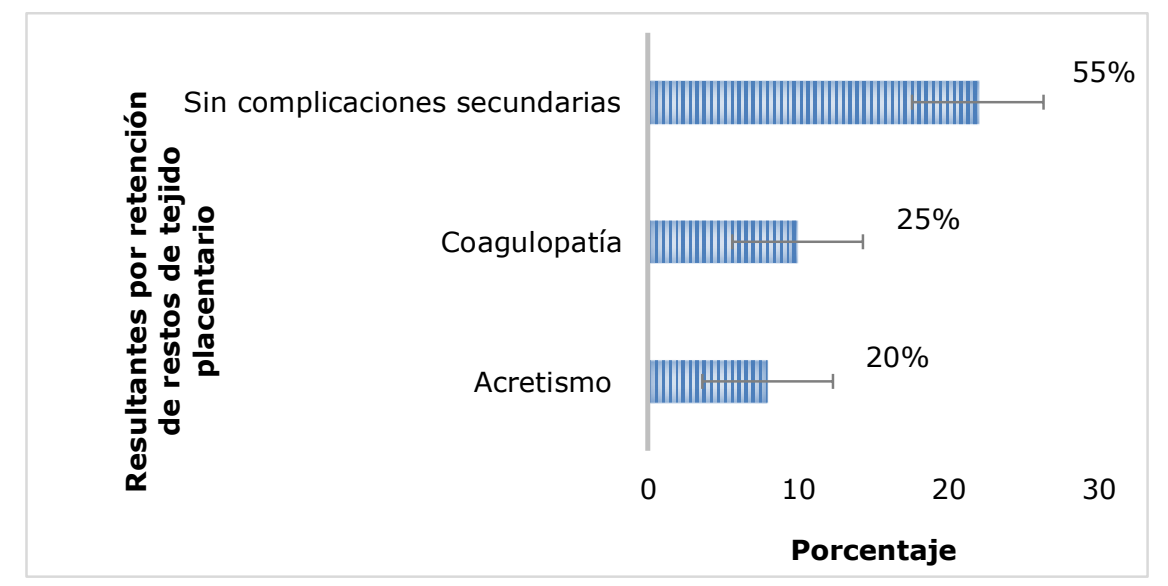

Figura 3: Patología asociada a la hemorragia postparto, por retención de restos placentarios.

\section{DISCUSIón}

La literatura especializada señala que la HPP sucede en los países en desarrollo (8), coincidiendo con el estudio realizado en el Hospital Gineco Obstétrico de Guayaquil ya que el número de pacientes es muy elevado (160 en dos años). Sobre los factores de riesgo de la $\mathrm{HPP}^{(10)}$ señalan que entre las causas más frecuentes de la HPP se encuentra la atonía uterina como la más frecuente, siendo responsable del 50 al $70 \%$ de las hemorragias del alumbramiento, sin embargo, en el presente estudio, fue el desgarro del suelo pélvico el que se presentó en mayor porcentaje, llegando a un $48,75 \%$ por sobre un $25 \%$ de casos de HPP por atonía. El mismo estudio ${ }^{(10)}$ indica que el desgarro pélvico puede deberse a la instrumentación empleada en el parto, no logrando comprobar esta particularidad en el estudio, debido a que no se obtuvo acceso a informes de tal profundidad.

Es menester resaltar que el estudio realizado no fue de intervención, sino, de revisiones de las historias clínicas, en ellas no se señalan el porqué de los desgarros del suelo pélvico, sobre este caso Abreu Pérez et al. ${ }^{(2)}$ indican que puede deberse a la fisiopatología multifactorial, la alteración de los músculos elevadores del ano y del núcleo fibroso del periné, por lo tanto, se conmina a realizar estudios sobre el objeto en particular.

El estudio de las hemorragias postparto inmediato tuvo sus limitaciones que se trataron de salvar de la mejor forma, uno de los más difíciles fue el hermetismo que existe en el departamento de archivos clínicos del hospital, a pesar de tener el consentimiento de la 
autoridad competente, no se brindó las facilidades para la obtención de los datos de las historias clínicas de las pacientes.

Los resultados de esta investigación son muy importantes para los especialistas en atención del parto en los hospitales de la región, se debe tomar en consideración los mismos, ya que permiten tener una cosmovisión más amplia del fenómeno de las HPP, e invitan a los profesionales de la atención al parto, a tomar en cuenta el de desgarro del suelo pélvico como factor resultante de los resultantes maternos.

Estos resultados también servirán de base al planteamiento de otro problema que se evidenció, cual es el desgarro del suelo pélvico en las pacientes evaluadas mediante las historias clínicas. Se deja sentada la hipótesis para un estudio posterior de si la activación y entrenamiento del suelo pélvico reduce el riesgo de desgarro y hemorragia postparto inmediato.

\section{REFERENCIAS BIBLIIOGRAFICAS}

1. OMS. Recomendaciones de la OMS para la prevención y el tratamiento de la hemorragia posparto. Ginebra: OMS; 2014.

2. Buchillón RP, Ramírez WJR, Pérez YR, Martínez LRC, Díaz GD, Peláez ALI. Hemorragia posparto temprana en puérperas atendidas en el Hospital General Provincial Docente de Ciego de Ávila. MediCiego. 12 de mayo de 2017; 23 (4): 4-11.

3. Osorio C, Monica T. Hemorragia post partoatonía uterina. Grupo Educativo Universidad Privada de Ica (Internet). diciembre de 2015 (citado 28 de agosto de 2019); Disponible en: http://repositorio.upica.edu.pe/xmlui/handl e/123456789/88

4. Díaz Acosta $D$, Rodríguez Adams EM, Martínez Torres J del C, Alerm González AJ. Fecal incontinence in women of medium age. Quality of life and confrontation styles. Rev Cub de Med Fis y Rehab. 26 de diciembre de 2016;8(S1):25-36.

5. Organización Mundial de la Salud. Recomendaciones de la OMS para la prevención y el tratamiento de la hemorragia postparto /WHO Recommendations for the Prevention and Treatment of Postpartum Hemorrhage. Ginebra: World Health Organization; 2014.

6. Iglesias Castro JM, Urbina Delgadillo D. Factores de riesgo asociados a hemorragia post- parto, en las mujeres que fueron atendidas en el servicio de GinecoObstetricia, del HECAM, Matagalpa en el período 2010-2013. (Internet) (Tesis). (Managua): Universidad Nacional Autónoma de Nicaragua; 2016. Disponible en:

http://repositorio.unan.edu.ni/5192/1/607 4.pdf

7. Flores ER, Lúe $\mathrm{P}$, Alfonso C, CardonaMorán KG. Determinación de la eficacia sobre los lineamientos técnicos para la implementación del" Código Rojo" en el manejo de las hemorragias obstétricas en pacientes en edades de 20 a 30 años, ASA

II, atendidas en el Hospital Nacional de la Mujer" Dra. María Isabel Rodríguez" durante los meses de Enero a Octubre del 2016 (Doctoral dissertation, Universidad de El Salvador). 2017; Disponible en: http://ri.ues.edu.sv/16290/.

8. Organización Mundial de la Salud. Mortalidad materna (Internet). 2018 (citado 28 de agosto de 2019). Disponible en: https://www.who.int/es/newsroom/fact-sheets/detail/maternal-mortality

9. INEC. Estimación de la razón de mortalidad materna en Ecuador. Quito; 2017.

10. Chandraharan E K. Diagnosis and management of postpartum haemorrhage. The BMJ. 2017; 358(358).

11. Solari AA, Solari GC, Wash FA, Guerrero GM, Enríquez GO. Hemorragia del postparto. Principales etiologías, su prevención, diagnóstico y tratamiento. Revista Médica Clínica Las Condes, 2014; 25(6): 993-1003. Disponible en: https://goo.gl/JUFk7g

12. Abreu $P$, Martínez $T C$, Rodríguez Adans EM, Alerm Gónzalez A, García Delgado JÁ. Prolapso de órganos pélvicos en la mujer. Revisión bibliográfica. Revista Cubana de Medicina Física y Rehabilitación. 2016; 99110.

13. Ecuador. Ministerio de Salud Pública. Prevención, diagnóstico y tratamiento de la hemorragia postparto. (base de datos en línea) Quito: MSP; 2013. Disponible en: https://www.salud.gob.ec/wpcontent/uploads/2016/09/Gu\%C3\%ADade-hemorragia-postparto.pdf

14. Asociación Médica Mundial. WMA - The World Medical Association-Declaración de Helsinki de la AMM - Principios éticos para las investigaciones médicas en seres humanos (Internet). 2017 (citado 28 de agosto de 2019). Disponible en: https://www.wma.net/es/policiespost/declaracion-de-helsinki-de-la-ammprincipios-eticos-para-las-investigacionesmedicas-en-seres-humanos/ 